\title{
Nonaffine rubber elasticity for stiff polymer networks
}

\author{
Claus Heussinger, Boris Schaefer, and Erwin Frey \\ Arnold-Sommerfeld Center for Theoretical Physics and Center for NanoScience, Department of Physics, Ludwig-Maximilians-Universität \\ München, Theresienstrasse 37, D-80333 München, Germany
}

(Received 11 April 2007; published 11 September 2007)

\begin{abstract}
We present a theory for the elasticity of cross-linked stiff polymer networks. Stiff polymers, unlike their flexible counterparts, are highly anisotropic elastic objects. Similar to mechanical beams, stiff polymers easily deform in bending, while they are much stiffer with respect to tensile forces ("stretching"). Unlike in previous approaches, where network elasticity is derived from the stretching mode, our theory properly accounts for the soft bending response. A self-consistent effective medium approach is used to calculate the macroscopic elastic moduli starting from a microscopic characterization of the deformation field in terms of "floppy modes"low-energy bending excitations that retain a high degree of nonaffinity. The length scale characterizing the emergent nonaffinity is given by the "fiber length" $l_{f}$, defined as the scale over which the polymers remain straight. The calculated scaling properties for the shear modulus are in excellent agreement with the results of recent simulations obtained in two-dimensional model networks. Furthermore, our theory can be applied to rationalize bulk rheological data in reconstituted actin networks.
\end{abstract}

DOI: 10.1103/PhysRevE.76.031906

PACS number(s): 87.16.Ka, 62.25.+g, 81.05.Lg

\section{INTRODUCTION}

The elasticity of flexible polymer gels is successfully described by the theory of rubber elasticity [1]. It ascribes the resistance to deformation to a reduction of conformational entropy induced by a changing end-to-end distance of individual polymer strands. In the classic approach, developed by Kuhn and others [2], the magnitude of the deformation of a single constituent polymer is usually assumed to derive from the macroscopically induced strain in an affine way. With this assumption the network problem is reduced to calculating the response of a single chain. In this sense, affine deformations represent a mean-field assumption that neglects spatial correlations and therefore the coupling between the network structure ("architecture") and the mechanical properties of its constituents.

In recent years, a different class of cross-linked networks made of semiflexible or stiff polymers have gained widespread interest. Their importance for biological systems as the cytoskeleton or extracellular matrix makes understanding their properties highly rewarding [3]. Out of the variety of biological stiff polymers, $F$-actin has emerged as a model system, which allows precise in vitro rheological measurements, for example in determining the (complex) frequencydependent shear modulus $G(\omega)$ and in particular its elastic component, the plateau modulus $G_{0}$ at intermediate frequencies. In these experiments various types of cross-linking proteins are being used [4-7] and the influence of the degree of cross-linking on the elastic modulus is investigated.

Stiff polymers, unlike their flexible counterparts, are highly anisotropic in their elastic response and may be characterized in terms of two qualitatively different deformation modes (see Fig. 1) [8,9]. The linear response to longitudinal forces acting parallel to the contour (stretching or compression), is due to the presence of thermally excited undulations similar to the (isotropic) stiffness of flexible polymers. The resulting effective spring constant of a stiff polymer of con- tour length $l_{s}, \quad k_{\|} \sim l_{p} / l_{s}^{4}$, depends on the temperaturedependent persistence length $l_{p} \sim T^{-1}$, which indicates the entropic origin. On the other hand, the resistance of the polymer to transverse forces (bending) is predominantly an energetic effect, leading to an increase in energy rather than to a decrease in entropy. Subsequently, the corresponding spring constant $k_{\perp} \sim l_{s}^{-3}$ is independent of temperature.

The presence of two elementary deformation modes complicates, but also enriches, the theoretical analysis of stiff polymer networks since it is not obvious which of the modes, or combination thereof, will dominate the macroscopic elastic response $[10,11]$. Leaving aside these difficulties, recent approaches $[9,12,13]$ have still adopted straightforward extensions of rubber elasticity to stiff polymer networks by assuming affine deformations to be present down to the scale of the individual polymer segment- the part of a polymer filament that connects two neighboring cross-links (see

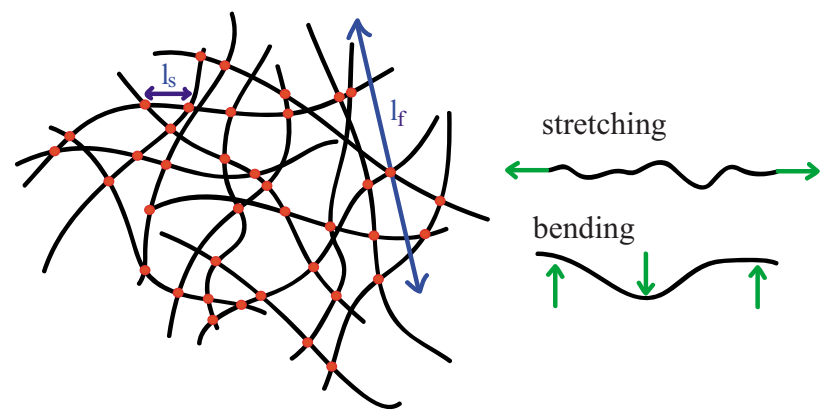

FIG. 1. (Color online) Sketch of a stiff polymer network with filaments that are straight on a scale $l_{f}$ and where the distances between crosslinks ("polymer segments") on a given filament are denoted by $l_{s}$. The response of the constituent stiff polymers to external forces is anisotropic with spring constants $k_{\|}$and $k_{\perp}$, characterizing their resistance to stretching and bending deformations, respectively. 
Fig. 1). In these models no bending deformations are present, leaving the stretching mode as the only possible source of elasticity.

In many systems of interest, however, the large value of the persistence length $l_{p} / l_{s} \gg 1$ calls this affine approach into question. This separation of length scales implies that the bending mode is in fact soft as compared to the stretching mode, since $k_{\|} / k_{\perp} \sim l_{p} / l_{s} \gg 1$. One would therefore expect the elastic energy to be dominated by low-energy bending deformations instead of highly expensive stretching modes [14]. Interestingly, recent simulations on random fibrous networks have shown that this is not always the case $[10,11,15-17]$. There, it was found that in networks with infinitely long filaments (for fixed density) the soft bending mode is suppressed and the elastic modulus is entirely given in terms of the stiffer stretching mode, similar to springs connected in parallel. In contrast, the same simulations performed in the more realistic situation of finite filament length have indeed identified an elastic regime that is dominated by soft bending deformations. The filament length thus strongly influences the elastic properties and is crucial for understanding the observed behavior. The affine theory, by working on the smaller scale of the polymer segments, is incapable of accounting for these effects.

In the present article, we expand on our recent publication [18] to develop an elastic theory that works on the scale of the whole polymer filament. The theory naturally explains the presence of a bending dominated regime as well as its suppression with increasing filament length. It is expected to be applicable to a broad class of filamentous networks with a soft bending mode. Similar to the classic theory of rubber elasticity it assumes that the cross-links adjust to the macroscopically applied strain without showing thermal fluctuations. In contrast to rubber elasticity, however, the cross-link movements are chosen such that the polymer end-to-end distances are kept unchanged. This automatically avoids energetically highly expensive stretching deformations and results in elastic moduli that derive from the soft bending mode only.

In the following, we assume that stiff polymers, characterized by $k_{\|} / k_{\perp} \gg 1$, effectively behave as if they were strictly inextensible bars, i.e. having infinite stretching stiffness $k_{\|} \rightarrow \infty$. Subsequently, we construct sets of "admissible" cross-link displacements that respect this inextensibility and thus retain a highly non-affine character. These displacement modes are referred to as "floppy modes" [19], highlighting the fact that in an equivalent network of central force springs they would carry no energy. Here, the finite bending stiffness of the polymers associates an elastic energy to each mode, which we use to calculate the macroscopic elastic constants of the network.

Section II will be concerned with the analysis of networks in the limit of diverging stretching stiffness $k_{\|} \rightarrow \infty$, which allows us to treat stiff polymers as inextensible bars. We will introduce the concept of the floppy modes and give an explicit construction valid for a broad class of network architectures.

In Sec. III we discuss the energy involved with exciting floppy modes in networks of stiff polymers, characterized by a finite, but soft, bending stiffness. Specifically, we will de- velop a theory that allows to calculate the network elastic constants in a self-consistent manner.

Section IV is devoted to the specific architecture of random fibrous networks in a planar geometry (two dimensonal), where we check our ideas against simulations.

\section{FLOPPY MODES}

Here, we are concerned with some general properties of networks of inextensible bars, so-called frameworks. While the bars are assumed perfectly rigid, they are allowed to freely rotate at the cross-links ("vertices"). In effect, both the stretching and the bending mode are eliminated, which leaves us with a purely geometric problem. By applying methods of rigidity theory [19] we will find that polymer networks when viewed as frameworks are not rigid and possess zero-energy deformation modes ("floppy modes"), for which we will give an explicit geometric construction. These modes, which may be viewed as the analog of the zeroenergy shear modes of regular square lattices, characterize the deformation field of the network under external strain. By accounting for the finite bending stiffness of the polymers, they are used to calculate the elastic energy stored in polymer networks and thus the elastic moduli.

\section{A. Maxwell counting}

It has first been realized by Maxwell [20] that a framework, consisting of $v$ vertices and $b$ bars, can undergo a transition from a floppy to a rigid state by increasing the coordination number $z$. Assuming that each bar represents an independent constraint for the total of $d v$ degrees of freedom in $d$ spatial dimensions, Maxwell derived the condition $b$ $-d v=0$ determining the rigidity transition. As the number of vertices can be rewritten in terms of the coordination number as $v=2 b / z$, this immediately yields a critical coordination of $z_{c}=2 d$. According to this simple Maxwell counting rule, frameworks are rigid, whenever their vertices have more than $z_{c}$ neighbors, while they will be floppy and allow for internal rearrangements otherwise.

With regard to stiff polymer networks this transition may be used to set up a classification where the elastic energy is dominated by either bending or stretching modes. While for $z<z_{c}$ bending modes can stabilize the otherwise floppy (zero-energy) central-force network, they only provide minor contributions to the energy once $z>z_{c}$. The honeycomb lattice in $2 \mathrm{D}$, for example, has a coordination of $z=3$ and is therefore bending dominated, while the triangular lattice with $z=6$ is clearly rigid and therefore stretching dominated. Imposing a deformation necessarily leads to the stretching of bonds. A particular case is the square lattice in two dimensions, which has precisely the critical coordination $z=z_{c}=4$. Although being floppy with respect to shear deformations, the network may be stabilized by introducing suitable boundary constraints or by adding additional bars along the diagonals of some of the squares. It turns out that in the limit of infinite system size a vanishing fraction of diagonal bars is needed to stabilize the network [21].

Maxwell-counting is only approximate, since one can always add redundant bars that do not constrain any degrees of 
freedom. This effect is taken into account by the modified Maxwell relation $b-d v=s-m$ [22]. In this picture redundant bars create overconstrained regions where a total of $s$ states of self-equilibrated internal stresses may exist. In general, a state of self-stress is defined as a set of bar tensions that is in static equilibrium with zero external force applied. At the same time underconstrained regions arise that allow for $m$ zero energy deformation modes, i.e., internal rearrangements that can be accommodated without changing the lengths of any of the bars to first order in the magnitude of the imposed strain. These are usually referred to as mechanisms or floppy modes.

In principle, the floppy modes of a pin-jointed structure may be found by studying the kinematic matrix $\mathbf{C}$ which relates vertex displacements $\mathbf{d}$ to segment extensions $\mathbf{e}=\mathbf{C d}$ [23]. The kinematic matrix thus constitutes a linear relation between displacements and extensions, which is only true for infinitesimally small displacements. The entries to the matrix can then easily be identified by considering the extension of a single bar oriented (in two dimensions) at an angle $\phi$ to the horizontal. For given displacements $\mathbf{d}_{i}=\left(u_{i}, v_{i}\right)$ at the two vertices $i=1,2$ the extension is found as

$$
e=\left(u_{2}-u_{1}\right) \cos \phi+\left(v_{2}-v_{1}\right) \sin \phi .
$$

The floppy modes then correspond to those vertex displacements that do not lead to any extensions in the bars. This amounts to calculating the null-space of the matrix, i.e., $\mathbf{C d}_{0}=\mathbf{0}$.

An elementary but illustrative example of a bar/joint network (adopted from Ref. [24]) is the "chair" shown in Fig. 2(a). Having $b=4$ bars and $v=2$ vertices, Maxwell's counting rule would imply that the structure is marginally rigid. Actually, there is also one floppy mode $m=1$ as well as one state of self-stress $s=1$. The former corresponds to the (infinitesimal) movement of the horizontal bar forming the seat, while the latter corresponds to a tension in the two vertical bars making the back.

For regular systems it is sometimes possible to guess the modes. Consider, for the purpose of illustration, a honeycomb lattice in two dimensions, where a coordination of $z$ $=3$ implies $b-2 v=-v / 2$. There is, accordingly, half a floppy mode per vertex. These modes are most easily identified with shear deformations along lines of symmetry [Fig. 2(b)]. Probing the shear response of the honeycomb along a given direction will cause each of the $N$ layers of cells to be displaced by a small amount $\delta$, which eventually has to add up to the externally imposed deformation $\Delta=N \delta$. Thus, there is "sharing" of the deformations between the individual cells and each layer contributes a small amount to fulfilling the constraints imposed by the macroscopical strain field. In other words, the deformation field in the honeycomb lattice is affine down to the scale of the individual cell, which experiences deformations $\delta=\Delta / N \propto l_{\text {cell }}$ proportional to its own size.

Another possibility to construct the floppy modes of the honeycomb network is given by the librations of individual hexagons [25] [see Fig. 2(c)]. These librations are, in contrast to the shear displacements, localized modes that are confined to a single cell and its immediate surroundings.

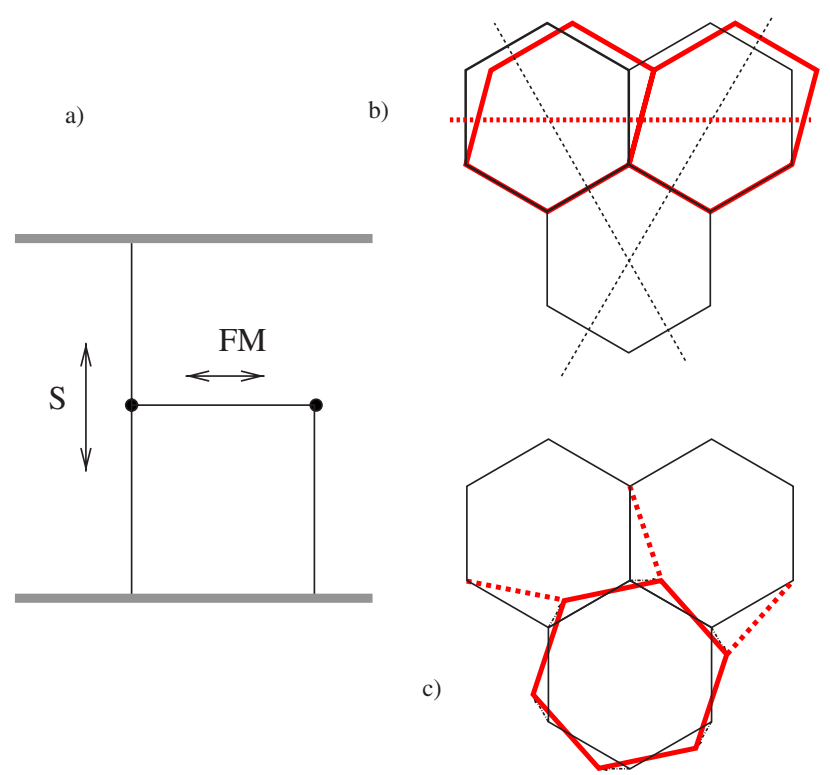

FIG. 2. (Color online) Illustration of the floppy modes. The "chair" in (a) has one floppy mode (FM) corresponding to the axial movement of the horizontal bar, as well as one state of self-stress $(S)$ located in the two vertical bars. The floppy modes of the honeycomb lattice may be constructed, (b) from the global shear deformations along any of the three dashed lines (as well as their parallels), or (c) from localized librations.

Since there is one libration per cell and each of the six corresponding vertices belongs to three cells, this also makes one mode for every two vertices.

\section{B. Floppy modes of stiff polymer networks}

Proteins used to cross-link stiff polymers into networks often have only two heads [26] such that there can only be two-, three- and four-fold connected vertices. The average coordination number in stiff polymer networks is therefore $z<4$, which would place it below the rigidity transition and render the network bending dominated.

In contrast to the very regular structures discussed above, stiff polymer networks are usually highly random. Nevertheless, as we will see below, the floppy modes can be constructed quite easily on scales $l_{f}$ over which the undeformed polymers can be assumed to be represented by straight fibers. For isolated polymers the length-scale $l_{f}$ can be identified with the persistence length $l_{p}$, while in networks the origin may be different and for example a consequence of the network generating process itself. It is the presence of the length-scale $l_{f}$ which renders the structure of stiff polymer networks qualitatively different from flexible polymer gels. The resulting fibrous appearance may be inspected in the figures of Refs. [27-29]. We have recently argued that the "fiber length" $l_{f}$ plays the role of the size of an effective unit cell [11], which in flexible polymer gels is set by the meshsize. In the following we use the word "fiber" in connection with the length $l_{f}$ over which the polymer remains straight. In later sections we will introduce a simple model system where fiber and polymer length are equal. 


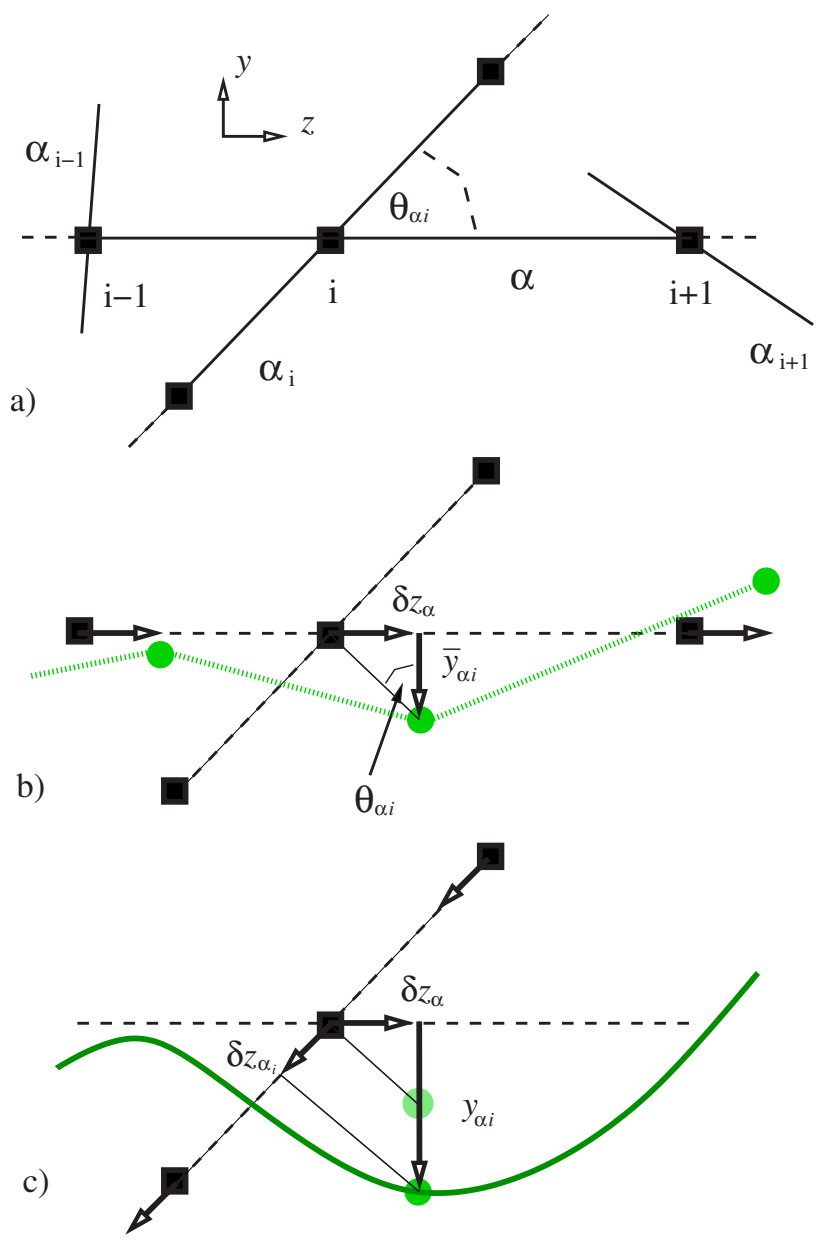

FIG. 3. (Color online) Construction of a floppy mode starting from the initial geometry as drawn in (a). In (b), the horizontal fiber is moved, while the surrounding fibers remain in their original positions. This leads to the new cross-link positions (green circles) with transverse deflection $\bar{y}_{\alpha, i}=-\cot \theta_{\alpha, i} \delta z_{\alpha}$ [Eq. (2)]. The component $\overline{\mathbf{y}}_{\alpha, i}^{\perp}$ (not drawn) is oriented perpendicular to the plane of the two fibers. In (c), the secondary fiber is also moved, such that the cross-link is now deflected according to $y_{\alpha, i}=\bar{y}_{\alpha, i}+\delta z_{\alpha_{i}} / \sin \theta_{\alpha, i}$ [Eq. (5)]. The solid green line represents the actual contour of the deformed fiber obtained by minimizing the bending energy along the entire fiber [see Eq. (4)].

Consider [see Figs. 3(a)-3(c)] a single (primary) fiber $\alpha$ of length $l_{f}$, which may be part of a longer polymer. It is imbedded into a network of other fibers, which we call secondary fibers, if they intersect the primary fiber at one of its cross-links $i=1, \ldots, n_{c l}$. The floppy-mode construction proceeds in two stages during which only the cross-links on the primary fiber are being moved. The rest of the network, in particular the neighboring filaments, will remain static such that the floppy mode stays highly localized similar to the librations of the hexagons discussed above. In the first step, we perform an arbitrary axial displacement $\delta z_{\alpha}$ of the primary fiber $\alpha$ as a whole. The axial movement of the crosslinks pertaining to this fiber induces a change in length of all neighboring segments on the crossing secondary fibers.

In the second step, therefore, one has to account for the length constraints on these segments by introducing cross- link deflections $\overline{\mathbf{y}}_{\alpha, i}$ transverse to the primary fiber. It turns out that to first order in $\delta z_{\alpha}$ all segment lengths can be kept at their repose length by choosing

$$
\overline{\mathbf{y}}_{\alpha, i}=-\delta z_{\alpha} \cot \theta_{\alpha, i} \hat{\mathbf{e}}_{\alpha, i}+\overline{\mathbf{y}}_{\alpha, i}^{\perp},
$$

where $\theta_{\alpha, i}$ is the angle between the two crossing fibers $\alpha$ and $\alpha_{i}$ at cross-link $i$. We denote by $\hat{\mathbf{e}}_{\alpha, i}$ a unit vector transverse to the primary fiber lying in the plane spanned by the two fibers, and by $\overline{\mathbf{y}}_{\alpha, i}^{\perp}$ an arbitrary vector perpendicular to this plane.

We would like to emphasize that the construction only works for infinitesimal $\delta z_{\alpha}$, while finite displacements necessarily lead to changes in bond lengths and therefore to stretching of bonds. As will be explained in more detail in Sec. IV A this has dramatic consequences on the nonlinear elasticity of the network, leading to strong strain stiffening behavior.

The construction can be performed for any of the $\alpha$ $=1, \ldots, N_{f}$ fibers, such that precisely $N_{f}$ floppy modes are identified in this way [42]. For the mode localized around fiber $\alpha$ one may define a set of vectors $\mathcal{Y}_{\alpha}=\left(\overline{\mathbf{y}}_{\alpha, 1}, \overline{\mathbf{y}}_{\alpha, 2}, \ldots\right)$, where the deflections of all cross-links in the network are combined. With respect to the standard vector scalar product one can then show that the set of floppy modes $\left\{\mathcal{Y}_{\alpha}\right\}$ is linearly independent, however, not orthogonal. Since a given cross-link $i$ always belongs to two filaments at the same time, there is obviously a coupling between the two corresponding modes. By superposition of the different $\mathcal{Y}_{\alpha}$ one may construct properly orthonormalized modes that, however, will not be localized anymore, but rather be extended over the whole system similar to the shear-modes of the honeycomb lattice.

For the particular architecture of a random fiber network in two dimensions (2D), to be introduced below (see Sec. IV), we have obtained these orthornormal modes by performing a singular value decomposition of the compatibility matrix C. One of the modes is visualized in Fig. 4, where the black lines indicate the floppy-mode displacements of the cross-links. One remarkable property is the heterogeneous distribution of amplitudes $x$, which leads to polynomial tails in the probability distribution, $P(x) \sim x^{-3}$ (see Fig. 5). The exponent is a direct consequence of the random orientation of the filaments which induces a probability distribution of angles $\theta$ between two intersecting filaments, $P(\theta) \sim \sin \theta$ [43]. By a transformation of variables to the floppy mode deflection $\bar{y} \sim \cot \theta$ (and thus to $x$ ) one finds a distribution $P(\bar{y}) \sim \sin ^{3} \theta(\bar{y}) \rightarrow \bar{y}^{-3}$, where the latter limit corresponds to large $\bar{y} \gg 1$.

\section{BENDING ENERGY ASSOCIATED WITH FLOPPY MODES}

To calculate the macroscopic elastic constants of the polymer network we have to associate an elastic energy to the floppy mode excitations. This is achieved by taking into account the finite bending stiffness of the polymers. Quite generally, the bending energy (for weakly undulating contours) can be written as 


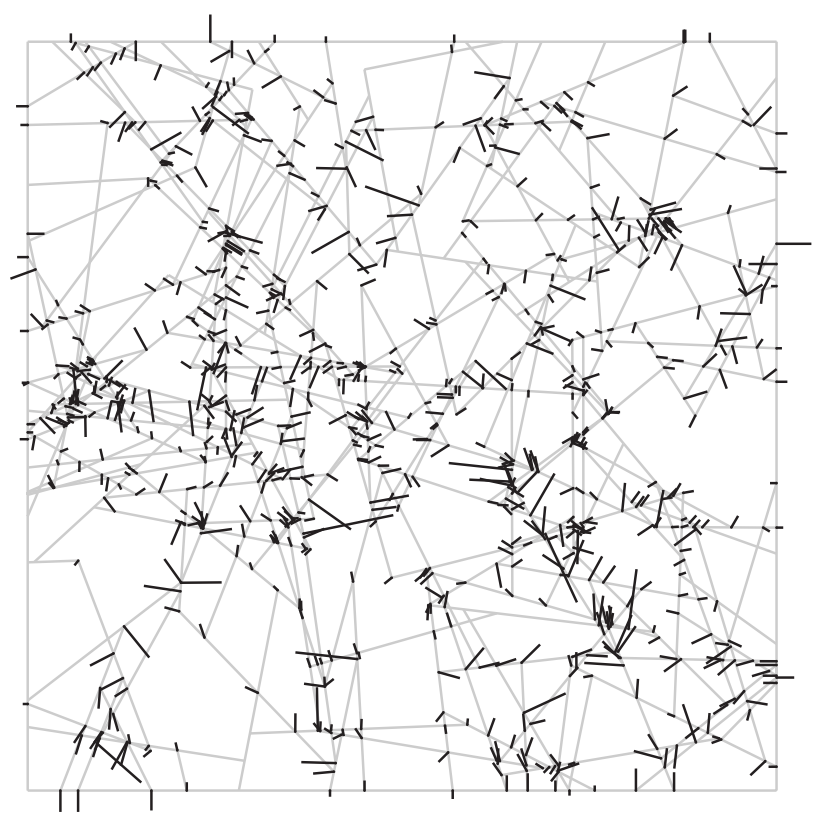

FIG. 4. Floppy mode of a random fiber network with 225 fibers (see Sec. IV). A fiber has the length of one third of the system size. Gray lines represent the network, black lines the floppy mode displacements. Note that the overall amplitude of the mode is arbitrary.

$$
W_{b}\left[\mathbf{y}_{\alpha}\right]=\frac{\kappa}{2} \int_{0}^{l_{f}}\left(\frac{d^{2} \mathbf{y}_{\alpha}}{d s^{2}}\right)^{2} d s
$$

where $\mathbf{y}_{\alpha}(s)$ denotes the transverse deflection at point $s$ along the backbone of polymer $\alpha$. The bending rigidity $\kappa$ is related to the persistence length by $\kappa=l_{p} k_{B} T$. The actual value of the energy contained in a floppy mode of amplitude $\delta z_{\alpha}$ can then be found by minimizing $W_{b}$ for a given set of cross-link positions $\mathbf{y}_{\alpha}\left(s_{\alpha, i}\right) \stackrel{!}{=} \overline{\mathbf{y}}_{\alpha, i}$ [taken from Eq. (2)], and gives

$$
W_{0}\left(\delta z_{\alpha}\right)=\min _{\mathbf{y}_{\alpha}(s), \mathbf{y}_{\alpha}\left(s_{\alpha, i}\right)=\overline{\mathbf{y}}_{\alpha, i}} W_{b}\left[\mathbf{y}_{\alpha}\right] .
$$

Technically, this is achieved by performing a cubic spline interpolation through the set of points $\left\{\left(s_{\alpha, i}, \overline{\mathbf{y}}_{\alpha, i}\right)\right\}_{i=1, \ldots, n_{c l}}$. This can be shown to be equivalent to the minimization of the bending energy in Eq. (3) [30]. As $d^{3} \mathbf{y}_{\alpha} / d s^{3}$ is proportional to the transverse force in the fiber, the discontinuities of the cubic spline in its third derivative reflect the external transverse force that is needed to keep the fiber in its deformed shape.

\section{A. Deformation field}

Up to now we have considered the movement of a single fiber in a static environment, where neighboring polymers remain fixed to their initial positions. To calculate the elastic modulus of a network, however, we have to take into account the fact that there is a different axial displacement $\delta z_{\alpha}$ for each of the $\alpha=1, \ldots, N_{f}$ fibers. We thus find [see Fig. 3(c)] that Eq. (2) has to be modified by a term $\delta z_{\alpha_{i}} / \sin \theta_{\alpha, i}$ due to the additional movement $\delta z_{\alpha_{i}}$ of the neighboring filament $\alpha_{i}$

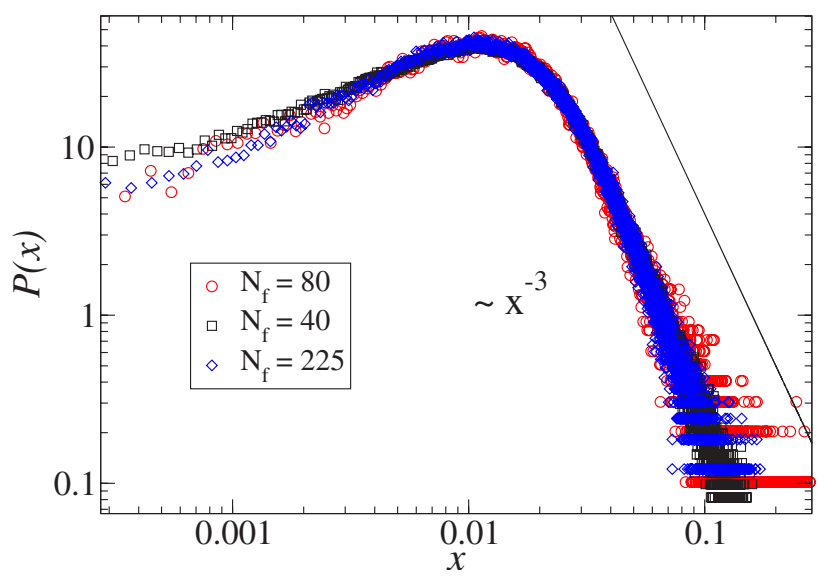

FIG. 5. (Color online) Normalized probability distributions of absolute values of floppy mode displacements, as shown in Fig. 4. The distributions for different fiber numbers $N_{f}$ can be rescaled on a single master curve by changing the overall amplitude of the modes.

at cross-link $i$. This amounts to the overall deflection

$$
\mathbf{y}_{\alpha, i}=\overline{\mathbf{y}}_{\alpha, i}+\frac{\delta z_{\alpha_{i}}}{\sin \theta_{\alpha, i}} \hat{\mathbf{e}}_{\alpha, i} .
$$

In principle, the modulus can now be found by minimizing the energy, consisting of contributions of the type of Eq. (4) from each of the $N_{f}$ fibers, with respect to the variables $\delta z_{\alpha}$ [44]. Compared to the original problem of having to minimize the energy with respect to the $N_{\mathrm{cl}} \sim N_{f} n_{\mathrm{cl}}$ cross-link coordinates, this is only a minimization with respect to $N_{f} \ll N_{\mathrm{cl}}$ variables. Still, this poses a challenging quenched disorder problem which can only be tackled numerically.

Here we further reduce the problem to an effective singlefiber theory, by making a simplifying assumption about the magnitude of the individual $\delta z_{\alpha}$. We assume that the fiber centers-of-mass $\mathbf{r}_{\mathrm{c} . \mathrm{m} .}^{\alpha}=\left(X_{\alpha}, Y_{\alpha}, Z_{\alpha}\right)$ follow the macroscopic strain field in an affine way, just as the centers of the hexagons did in the honeycomb lattice. This is equivalent to assuming that the displacement field is affine on the scale of the fiber length $l_{f}$. Note, however, that this does by no means imply that the elastic elements themselves undergo affine deformations, as will become clear below. For a given macroscopic shear $\gamma \equiv \gamma_{x y}$ we find $\delta \boldsymbol{r}_{\mathrm{c} . \mathrm{m} .}^{\alpha}=\gamma Y_{\alpha} \hat{\mathbf{e}}_{x}$ and thus

$$
\delta z_{\alpha}=\gamma Y_{\alpha} \cos \phi_{\alpha},
$$

which is just the projection of the affine displacement on the fiber axis, oriented at an angle $\phi_{\alpha} \in[-\pi / 2, \pi / 2]$ with respect to the $x$ axis. Using Eqs. (6) and (2) one can write Eq. (5) as

$$
\mathbf{y}_{\alpha, i}=-\delta z_{\text {rel }}^{\alpha} \cot \theta_{\alpha, i} \hat{\mathbf{e}}_{\alpha, i}+\overline{\mathbf{y}}_{\alpha, i}^{\perp},
$$

where we have defined

$$
\delta z_{\text {rel }}^{\alpha}=\gamma\left(Y_{\alpha} \cos \phi_{\alpha}-Y_{\alpha_{i}} \frac{\cos \left(\theta_{\alpha, i}+\phi_{\alpha}\right)}{\cos \theta_{\alpha, i}}\right) .
$$

Upon comparison of Eq. (7) with Eq. (2) one may interpret 
$\delta z_{\text {rel }}^{\alpha}$ as specifying the movement of the primary fiber relative to its surrounding. Note, however, that this relative displacement $\delta z_{\text {rel }}^{\alpha}$ depends on the orientations $\phi_{\alpha}$ and $\phi_{\alpha}+\theta_{\alpha, i}$ of the crossing filaments, as well as on the arc-length along the primary fiber (via $Y_{\alpha_{i}}$ ). In contrast to Eq. (2), which follows from moving the primary fiber in a fixed environment, Eqs. (7) and (8) are derived from a joint movement of all fibers.

For the following we are only interested in the typical magnitude of $\delta z_{\text {rel }}^{\alpha}$, which may be obtained by averaging over the angles $\phi_{\alpha}$ and estimating the typical distance between the center of masses of the intersecting fibers as $Y_{\alpha}-Y_{\alpha_{i}} \sim l_{f}$. We thus find that $\delta z_{\text {rel }}^{\alpha} \propto \gamma l_{f}$.

By assuming affine displacements of the fiber centers, we have thus succeeded in reducing the many-body problem of the movement $\delta z_{\alpha}$ of $N_{f}$ interacting fibers to the case of a single fiber moving the amount $\delta z_{\text {rel }}^{\alpha} \sim \gamma l_{f}$ relative to its surrounding. It should be made clear that this assumption is different from the usual approach of assigning affine deformations on the scale of the single polymer segment $[9,12,13]$. The latter would lead to deformations $\delta_{\text {aff }} \propto \gamma l_{s}$, proportional to the length $l_{s}$ of the segment. Instead, axial displacements of the fiber as a whole are, by construction of the floppy mode, directly translated into nonaffine deformations $\delta_{\text {na }} \propto \gamma l_{f}$, which do not depend on the length of the segment but rather on the scale of the fiber length $l_{f}$. It is worth pointing out the subtle difference between "affine displacements" of single points (the fiber centers-of-mass), and "affine deformations" of fiber segments of length $l_{s}$.

\section{B. Self-consistent effective medium theory}

The effective single fiber picture developed in the preceding section is particularly well suited to set up an effective medium approach that includes corrections to the affine assumption embodied in Eq. (6). For this let us consider the excitation of a localized floppy mode of magnitude $\delta z_{\alpha}$ $\sim \gamma l_{f}$ in a single fiber. The scaling with the fiber length $l_{f}$ indirectly reflects the affine displacement of all the fiber centers in the network.

The energy required to move this single fiber against a static environment is given by Eq. (4). Most importantly, however, this fiber energy may be reduced at the cost of deforming the surrounding, i.e. by spreading the mode to the neighboring filaments. The amplitudes of the so-generated secondary floppy modes may be found from solving Eq. (5) for $\delta z_{\alpha_{i}}$. We thus find

$$
\delta z_{\alpha_{i}}=\sin \theta_{\alpha, i}\left(\mathbf{y}_{\alpha, i}-\overline{\mathbf{y}}_{\alpha, i}\right) \cdot \hat{\mathbf{e}}_{\alpha, i}
$$

which highlights the fact that a secondary mode of amplitude $\delta z_{\alpha_{i}}$ occurs when the actual transverse deflection $\mathbf{y}_{\alpha, i}$ is different from the floppy-mode prescription $\overline{\mathbf{y}}_{\alpha, i}$. Furthermore, due to the scalar product with $\hat{\mathbf{e}}_{\alpha, i}$, the displacement $\mathbf{y}_{i}^{\perp}$ perpendicular to the plane defined by the two intersecting polymers does not contribute, $\mathbf{y}_{\alpha, i}^{\perp} \cdot \hat{\mathbf{e}}_{\alpha, i}=0$.

With Eq. (9) we find that Eq. (4) has to be modified by the bending energy contribution $W_{0}$ from the neighboring filaments giving

$$
W_{1}\left(\delta z_{\alpha}\right)=\min _{\mathbf{y}_{\alpha}(s)}\left(W_{b}\left[\mathbf{y}_{\alpha}\right]+\sum_{i=1}^{n_{\mathrm{cl}}} W_{0}\left(\delta z_{\alpha_{i}}\right)\right) .
$$

Unlike in Eq. (4), where the cross-link variables on the primary fiber were constrained to be $\mathbf{y}_{\alpha}\left(s_{\alpha, i}\right)=\overline{\mathbf{y}}_{\alpha, i}$, here they remain unconstrained and move such that the total energy, deriving from both primary and secondary fibers, is minimized. Note, however, that the deflections on the secondary fibers are still constrained and given by Eq. (2). This deficiency can be cured by taking into account further levels of filaments (tertiary, ...), thus defining a sequence of energies $\left(W_{0}, W_{1}, W_{2}, \ldots W_{\infty}\right)$, the fixed point of which is found by substituting on both sides of Eq. (10) one and the same asymptotic function $W_{\infty}$.

Since the resulting expression still depends on the quenched random network structure in a complicated way, we have recently proposed an effective medium approximation that uses the averaged $\left\langle\left[W_{\infty}\right]\right\rangle \equiv W$ instead [18]. For reasons that will immediately become apparent we have defined two averaging procedures. The angular brackets $\langle$.$\rangle denote$ averaging over the random variables on the primary fiber, the cross-link positions $s_{\alpha, i}$ and angles $\theta_{\alpha, i}$. The probability distributions of these variables provide the most important characterization of the architecture of the network. The brackets [.] denote averaging with respect to the remaining randomness in the subsequent hierarchies of fibers. Mathematically, the effective medium approximation is implemented by interchanging this latter average with the minimization operation. Physically, this amounts to assuming that one and the same medium $(W)$ is felt by all the cross-links on the primary fiber. One thus arrives at the final equation

$$
W\left(\delta z_{\alpha}\right)=\left\langle\min _{y_{\alpha}(z)}\left(W_{b}\left[y_{\alpha}\right]+\sum_{i=1}^{n_{\mathrm{cl}}} W\left(\delta z_{\alpha_{i}}\right)\right)\right\rangle,
$$

where $\delta z_{\alpha_{i}}$ is given by Eq. (9). In principle Eq. (11) has to be solved self-consistently for the function $W(x)$. Since we are concerned with small displacements only the energy may be expanded to harmonic order as $W(x)=k x^{2} / 2$, which gives

$$
W\left(\delta z_{\alpha_{i}}\right)=\frac{1}{2} k \sin ^{2} \theta_{\alpha, i}\left(y_{\alpha, i}-\bar{y}_{\alpha, i}\right)^{2},
$$

where we defined $y_{\alpha, i}=\mathbf{y}_{\alpha, i} \cdot \hat{\mathbf{e}}_{\alpha, i}$ and similar for $\bar{y}_{\alpha, i}$. With this parametrization Eq. (11) has to be solved for the single unknown parameter $k$.

Equation (11) can be interpreted as follows. The energy associated with the excitation of a single floppy mode of amplitude $\delta z$ has two contributions. The first term, corresponding to the bending energy of the primary filament, $W_{b}$, dominates if the cross-links follow the floppy-mode prescription and $\delta z_{\alpha_{i}} \approx 0\left(y_{\alpha, i} \approx \bar{y}_{\alpha, i}\right)$. On the other hand, the energy is mainly stored in the surrounding medium if the cross-links deviate strongly from the floppy mode, $y_{\alpha, i} \approx 0$, in which case the bending energy vanishes, $W_{b} \approx 0$. Since medium deformations can only occur in the form of floppy modes, the stiffness $k$ of the medium is the same as the stiffness of the fiber. This allows to solve the equation self-consistently, which can easily be done numerically as will be explained in 


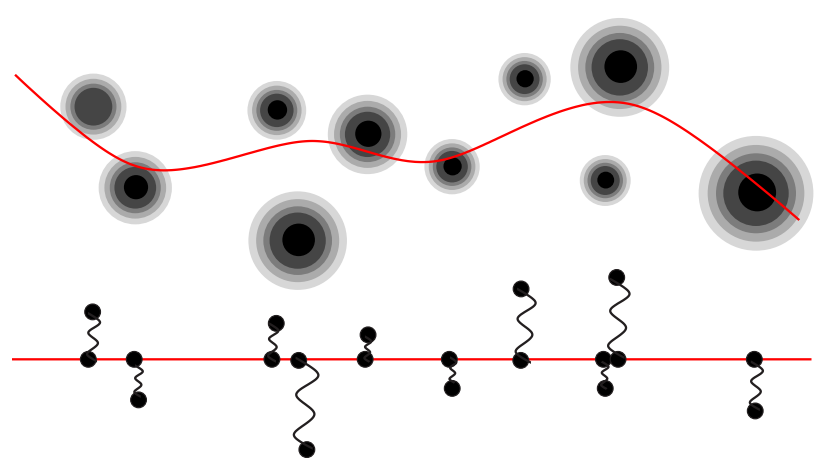

FIG. 6. (Color online) The energy in Eq. (11) is that of a stiff polymer attached to springs of variable stiffness. It may therefore be interpreted as a polymer in a random potential. The potential is attractive and localized at pinning sites given by Eq. (2).

the appendix. There, we will also solve Eq. (11) for some exemplary network structures. Note that the elastic modulus $G$ of the entire network can easily be obtained from the single-fiber energy $W$ by equating $G \gamma^{2} \sim N_{f} W$.

It is worth mentioning that Eqs. (11) and (12) may be interpreted as the zero temperature limit (or the saddle-point approximation) to a fluctuating stiff polymer in a random array of harmonic pinning sites with stiffnesses given by $k \sin ^{2} \theta_{\alpha, i}$ (see Fig. 6). Compared to the "bare floppion" defined by Eqs. (2)-(4), the excitation given by Eq. (11) is "dressed" and incorporates the interactions with the medium on a Cayley-tree level.

\section{RANDOM NETWORK IN 2D}

Having presented the general concepts we now proceed to introduce a simple model system where the ideas may be tested. The random two-dimensional network, the "Mikado model," has the advantage that it only needs one structural parameter, the density of fibers $\rho$.

The network is defined by randomly placing $N$ elastic fibers of length $l_{f}$ on a plane of area $A=L^{2}$ such that both position and orientation are uniformly distributed [31-33]. The fiber-fiber intersections are assumed to be perfectly rigid, but freely rotatable cross-links that do not allow for relative sliding of the filaments. The randomness entails a distribution of angles $\theta \in[0, \pi]$ between two intersecting filaments

$$
P(\theta)=\frac{\sin \theta}{2},
$$

while distances between neighboring intersections, the segment lengths $l_{s}$, follow an exponential distribution [34]

$$
P\left(l_{s}\right)=\left\langle l_{s}\right\rangle^{-1} e^{-l_{s}\left\langle l_{s}\right\rangle} .
$$

The mean segment length $\left\langle l_{s}\right\rangle$ is inversely related to the line density $\rho=N l_{f} / A$ as $\left\langle l_{s}\right\rangle=\pi / 2 \rho$. The segments are modeled as classical beams with cross-section radius $r$ and bending rigidity $\kappa$. Loaded along their axis ("stretching") such slender rods have a rather high stiffness $k_{\|}\left(l_{s}\right)=4 \kappa / l_{s} r^{2}$, while

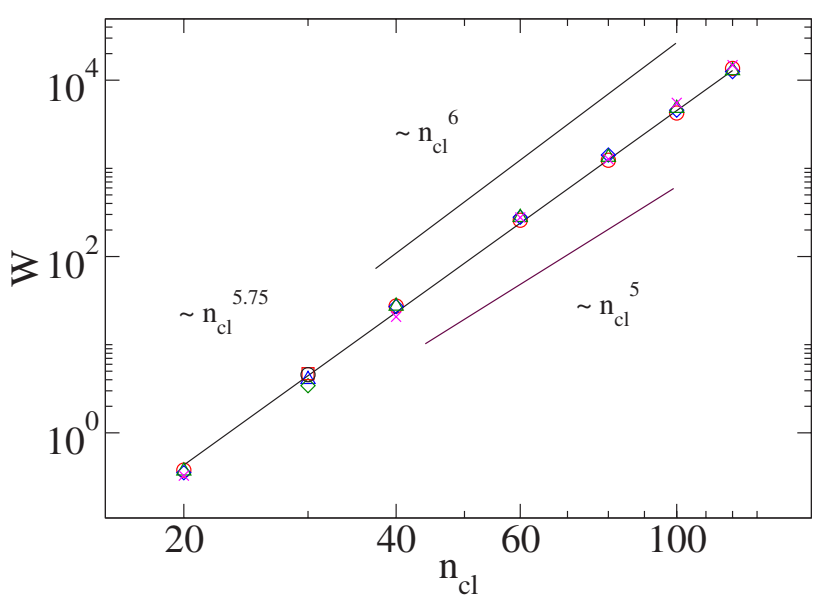

FIG. 7. (Color online) Solution of Eqs. (11) and (12) for various numbers $n_{\mathrm{cl}}$ of cross-links per filament. The randomness is defined by Eqs. (13) and (14). The different symbols at given $n_{\mathrm{cl}}$ relate to ensembles of varying size $N=100 \ldots 1000$. The lines $W \sim n_{\mathrm{cl}}^{5}$ and $W \sim n_{\mathrm{cl}}^{6}$ serve to illustrate the quality of the fit.

they are much softer with respect to transverse deformations $k_{\perp}\left(l_{s}\right)=3 \kappa / l_{s}^{3}$ ("bending").

Numerical simulations [15-17] for the effective shear modulus $G$ of this network have identified a crossover scaling scenario characterized by a length scale

$$
\xi=l_{f}\left(\delta \rho l_{f}\right)^{-\nu}
$$

with $\nu \approx 2.84[16,45]$ that mediates the transition between two drastically different elastic regimes. For fiber radius $r \gg \xi$ the system is in an affine regime where the elastic response is mainly dominated by stretching deformations homogeneously distributed throughout the sample. The modulus in this regime is simply proportional to the typical stretching stiffness, $G_{\text {aff }} \propto k_{\|}\left(\left\langle l_{s}\right\rangle\right)$ and independent of the fiber length $l_{f}$. This is in marked contrast to the second regime at $r \ll \xi$. There, only non-affine bending deformations are excited and the modulus shows a strong dependence on fiber length,

$$
G_{\mathrm{na}} \propto k_{\perp}\left(\left\langle l_{s}\right\rangle\right)\left(\frac{l_{f}}{\left\langle l_{s}\right\rangle}\right)^{\mu-3},
$$

and thus on density, $G_{\text {na }} \propto \delta \rho^{\mu}$ where $\mu=2 \nu+1 \approx 6.67$.

As this latter non-affine regime is characterized by a ratio $k_{\|}\left(\left\langle l_{s}\right\rangle\right) / k_{\perp}\left(\left\langle l_{s}\right\rangle\right) \sim\left(\left\langle l_{s}\right\rangle / r\right)^{2} \gg 1$, and therefore a bending mode that is soft as compared to the stretching mode, we may apply the floppy-mode picture developed in previous sections to calculate the exponent $\mu$. To this end, we numerically solve Eq. (11) for varying numbers $n_{\mathrm{cl}} \sim \rho l_{f}$ of crosslinks per fiber. The average $\langle$.$\rangle is thereby defined in terms of$ the probability distributions of Eqs. (13) and (14). As a result, we find the fiber energy $W$ to depend on density $\rho$ as $W \sim \rho^{x}$ and $x \approx 5.75 \pm 0.1$ (see Fig. 7). The shear modulus can be inferred from $W$ as $G \sim\left(\rho l_{f}\right) W \sim \rho^{6.75}$, which reproduces the exponent $\mu$ as measured in the simulation to a remarkable accuracy. 
Based on the formalism of the preceding sections we have also developed [18] a scaling argument that allows approximate solution of Eq. (11) in terms of a single length-scale $l_{\min }$, which on a microscopic scale governs the coupling of the fiber to the matrix. Since the stiffness $k_{\perp} \sim \kappa / l_{s}^{3}$ of the individual polymer segment is strongly increasing with decreasing its length $l_{s}$, we assume that segments with $l_{s}$ $<l_{\text {min }}$ rather deform the surrounding medium than being deformed itself, while longer segments $l_{s}>l_{\min }$ are not stiff enough to deform the medium. The scale $l_{\min }$ therefore plays the role of a minimal length below which segments are stiff enough to remain undeformed.

In terms of the cross-link deflections $y_{i}$, this implies that long (and soft) segments have $y_{i} \approx \bar{y}_{i}$, while short (and stiff) segments have cross-links that are in their original position $y_{i} \approx 0$. Since the energy of a segment of length $l_{s}$ can be written as $w\left(l_{s}\right) \sim k_{\perp} y_{i}^{2} \sim \kappa y_{i}^{2} / l_{s}^{3}$, we find that the elastic energy is reduced by the amount $w\left(l_{\min }\right) \sim \kappa \bar{y}_{i}^{2} / l_{\min }^{3}$ as compared to the situation where also the short segments are deformed. In turn, the energy in the neighboring fiber is increased, where a floppy mode of amplitude $\delta z \sim \bar{y}_{i}$ is excited. The length scale $l_{\min }$ can therefore be determined by equating the energy reduction in the small segments, $w\left(l_{\min }\right)$, with the energy increase due to the additional floppy mode in the neighboring fiber. This latter contribution can be calculated as an average over all segments of length $l_{s}>l_{\min }$ thus giving

$$
W \simeq n_{\mathrm{cl}} \int_{l_{\min }}^{\infty} d l_{s} P\left(l_{s}\right) w\left(l_{s}\right)^{!} \stackrel{!}{=} w\left(l_{\min }\right) .
$$

As a result, we find $l_{\min } \simeq 1 / \rho^{2} l_{f}$ and thus for the average fiber energy $W \simeq \kappa\left(\rho l_{f}\right)^{6} / l_{f}$. This corresponds to an exponent $\mu=7$, which confirms the previous analysis.

From Eq. (14) one may also induce a probabilistic interpretation of the length-scale $l_{\min }$. Segments with lengths $l_{s}$ $<l_{\min }$ will occur on average only once along a given fiber. This may be seen from solving the equation

$$
\int_{0}^{l_{\min }} d l_{s} P\left(l_{s}\right) \sim \frac{1}{n_{\mathrm{cl}}}
$$

stating that small segments will occur once in every $n_{\mathrm{cl}}$ $\sim \rho l_{f}$ cross-links. There will, therefore, typically be one segment per fiber in the undeformed configuration $y_{i} \approx 0$, while all others follow the floppy mode.

One may actually use these results to relate the lengthscale $\xi$ found in the simulation to $l_{\min }$ by $\xi \sim l_{f}\left(l_{\min } / l_{f}\right)^{3 / 2}$. We therefore propose that the scaling variable $x=r / \xi$ of Eq. (2) in [16] is written in the alternative form $x^{-2}$ $\sim k_{\|}\left(l_{f}\right) / k_{\perp}\left(l_{\min }\right)$ reflecting the more fundamental nature of the length scale $l_{\text {min }}$. Written in this way $x^{-2}$ directly encodes the relative stiffness of the deformation modes stretching and bending on the scale of the whole polymer fiber. The modulus then takes the scaling form

$$
G(r, \rho)=\rho^{\mu} g\left[k_{\|}\left(l_{f}\right) / k_{\perp}\left(l_{\min }\right)\right],
$$

which is the equivalent of Eq. (2) in [16].

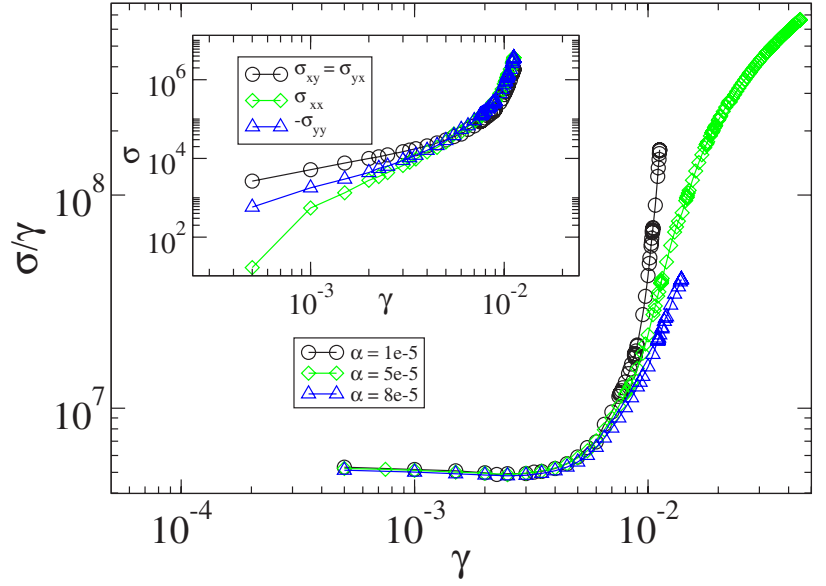

FIG. 8. (Color online) Nonlinear "modulus" $\sigma / \gamma$ in the bending dominated regime $\left(\rho l_{f}=30\right)$ for various values of the aspect ratio $\alpha=r / l_{f}$. Inset: The stress increases linearly up to a strain of about $1 \%$. Normal stresses quickly rise in magnitude and eventually are of the same order and proportional to the shear stress.

The only requirement for the presence of a bending dominated regime (beyond the scale separation $k_{\|} / k_{\perp} \gg 1$ ) is a low coordination number, which for the random fiber network can be calculated as $z=4\left[1-\left(\rho l_{f}\right)^{-1}\right]$. This places the network below the rigidity transition for any finite $l_{f}$, while increasing the filament length $l_{f} \rightarrow \infty$ the critical coordination of $z_{c}=4$ is asymptotically reached. As an implication the bending mode must eventually be suppressed.

The above analysis clearly shows that the proposed floppy mode concept can be utilized to understand the bending dominated elasticity in the random fibrous network. In addition, it allows to extract the length scale $l_{\min }$ that is ultimately responsible for the strong density dependence of the elastic modulus. It is important to note that other network structures in general will not necessarily feature the same length scale $l_{\min }$, even though the basic formalism of the floppy bending modes can still be applied. The exponents characterizing the elastic response will thus depend on network architecture, as is exemplified in the Appendix.

In Ref. [27] we have furthermore applied the theory to explain the mechanics of reconstituted actin networks, where filaments are cross-linked and bundled by fascin. By taking into account the fact that bundles have to be characterized by a length-dependent bending rigidity $\kappa(L)[35-37]$ it was possible to explain the observed dependence of the elastic modulus on actin and fascin concentration.

\section{A. Nonlinear elasticity arising from geometric effects}

Here, we report on additional simulations probing the nonlinear modulus of the structure. Note that in these simulations the material properties of the fibers remain linear, such that the nonlinearities result from geometrical effects only. As one can see from Fig. 8, the network is strongly stiffening already at very small values of strain.

Similar results have recently been reported in [38], where the stiffening behavior was attributed to a crossover from 
bending to stretching dominated elasticity. The floppy mode picture allows to give this cross over a microscopic explanation. As argued in Sec. II B, the floppy modes of the fibrous network are only adequate for infinitesimally small displacements $\delta z$. The construction embodied in Eq. (2) keeps segment lengths invariant to first order in $\delta z$ only, such that any finite deformation will necessarily lead to stretching of the bonds.

Note that this stiffening mechanism is not mediated by non linear material properties of the fibers but rather is of geometric origin and is due to the specific structural arrangement in the fibrous architecture. It is therefore of different nature than the stiffening mechanism inherent to single semiflexible polymers, where an applied tension can stretch the polymer only as far as there is stored length available [39].

In the nonlinear regime we have also measured the normal stresses $\sigma_{x x}$ and $\sigma_{y y}$ that act perpendicular to the principal strain direction. We found (see inset to Fig. 8) that these stresses can become of the order of the shear stresses $\sigma_{x y}$ and have a negative sign indicating that the network pulls in during the course of the deformation. A similar effect has recently been observed in rheological measurements on $F$-actin networks [40] and rationalized in terms of the highly nonlinear entropic stretching response of single polymers. Note that in our simulations the same effect occurs within a purely mechanical picture, where no material non linearities are present. It is explained with the fact that the additional amount of contour length necessary to undergo a finite floppy mode can only come from pulling in the fiber ends. This is equivalent to a network contraction which leads to the observed large normal stresses.

\section{B. Nonstraight fibers}

In real networks fibers will never be perfectly straight. We have argued above that in this case the scale of the fiber length $l_{f}$ must be viewed as the length scale over which the polymer remains straight. With this in mind our theory also holds for networks where fibers are nonstraight, as long as the undulation wavelength $\lambda \sim l_{f}$ is larger than the distance between cross-links $\bar{l}_{s}$.

In this section we investigate the effects of introducing undulations with wavelengths comparable to the cross-link distance, $\lambda \sim\left\langle l_{s}\right\rangle$. To this end we have manually generated zig-zag fibers by randomly displacing the cross-links by some maximal amount $\Delta \cdot l_{f}$. A similar analysis has been performed in Ref. [38], where a substantial decrease in the degree of nonaffinity of the deformation field has been found. Similarly, we find that the system develops a new crossover to a regime of affine bending deformations (see Fig. 9), where the modulus scales as $G \propto \delta \rho^{3}$, a behavior well known from bending dominated cellular foams $[11,14,41]$.

In this new regime the bending deformations come from pulling out the zig-zags similar to the pulling of thermally activated polymer undulations. We find that the curves may be scaled by using the same length scale $l_{\min } \sim \delta \rho^{-2}$ that served as a lower cutoff in segment lengths. The modulus thus takes the following scaling form

$$
G(\Delta, \delta \rho)=\Delta^{-\mu / 2} g\left(\Delta / l_{\min }\right),
$$

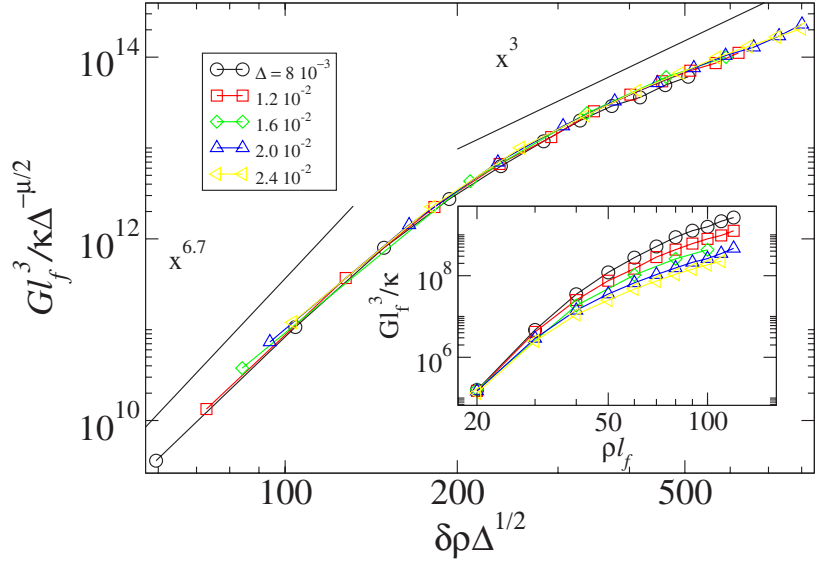

FIG. 9. (Color online) Shear modulus $G$ (inset) and scaling function $g$ of Eq. (20) for various values of $\Delta$. Collapse is achieved by plotting $G \Delta^{\mu / 2}$ as a function $x=\delta \rho \Delta^{1 / 2}$ and known exponent $\mu$ $=6.7$ [16]. The asymptotic regimes show the scaling properties of straight fibers, $g(x) \sim x^{6.7}$, and foams, $g(x) \sim x^{3}$, respectively.

where the scaling function has the limiting form $g(x \ll 1)$ $\sim x^{\mu / 2}$ to eliminate the $\Delta$ dependency. For large values of the scaling variable $x \gg 1$ we have to recover the scaling properties characteristic for foams, giving $g(x \gg 1) \sim x^{3 / 2}$. This analysis highlights once more the fundamental role played by the length scale $l_{\min }$ in establishing the elastic response of the network. Here, it acts as a crossover scale, that mediates the transition to a foamlike bending regime at strong disorder $\Delta \gg l_{\min }$.

Note that, by introducing kinks in the contour of the fibers, the floppy modes start to spread beyond the single fiber to which they were confined originally. A kink is most conveniently characterized by the angle $\psi$ through which the direction of the fiber changes at the location of the kink. By displacing a cross-link by the amount $\Delta$ one thus finds for the angle $\sin \psi=\Delta / l_{s}$, where $l_{s}$ is the length of the segment that ends at the cross-link. Exciting the fiber with a floppy mode of amplitude $\delta z$, a finite kink-angle $\psi$ leads to the fraction $\delta z^{\prime} \sim \delta z \sin \psi \sim \delta z \Delta / l_{s}$ being coupled into the neighboring fiber. At the crossover, defined by $x=\Delta / l_{\min } \sim 1$, we therefore find that for a segment of length $l_{s}=l_{\min }, \delta z^{\prime}\left(l_{\min }\right) \approx \delta z$. In this situation the floppy mode is transmitted to the neighboring fiber without attenuation of its amplitude. Since segments of length $l_{\min }$ statistically occur once per filament, the crossover point also marks the onset of a complete delocalization of the floppy modes.

\section{CONCLUSION}

We started our discussion with the assumption that the elasticity of stiff polymer networks is governed by the action of the bending mode. This assumption is based on the recognition that in systems where the persistence length is large, bending as compared to stretching is by far the softer mode. The respective spring constants are scale-separated and obey the relation $k_{\|} / k_{\perp} \sim l_{p} / l \gg 1$. 


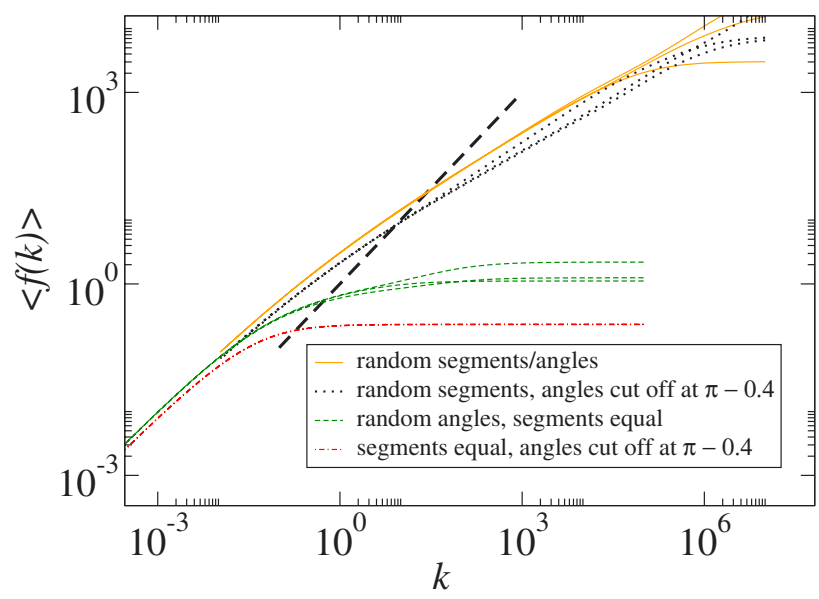

FIG. 10. (Color online) Graphical solution of Eq. (A2) for a fiber with $n_{c l}=40$ cross-links imbedded in networks of varying architectures. $\langle f(k)\rangle$ is plotted as a function of $k$ for ensembles of varying sizes. The solution to Eq. (A2) is found by intersecting a solid curve with the dashed (bisecting) line.

One immediate implication of this scenario is that polymer end-to-end distances have to stay constant, which necessitates deformations that are highly non-affine. We have characterized this non-affine deformation field by constructing the floppy modes of the structure [18]. These are defined as the set of cross-link displacements that do not lead to any stretching of bonds. With this microscopic deformation field it is possible to calculate the macroscopic elastic moduli on the level of a self-consistent effective medium theory that incorporates fiber-medium interactions within a Cayley-tree approximation.

As a result the anomalous scaling properties of the linear shear modulus as determined by computer simulations of two-dimensional random networks $[16,17]$ are explained. The exponents are found to be a consequence of the special architecture of the network that features two different length scales. On the mesoscopic scale the fiber length $l_{f}$ induces a non-affine deformation field, with segment deformations $\delta_{\text {na }}$ following the macroscopic strain $\gamma$ as $\delta_{\text {na }} \sim \gamma l_{f}$, instead of as $\delta_{\text {aff }} \sim \gamma l_{s}$, which would result from an affine deformation field. Microscopically, a second length $l_{\min }$ plays the role of a minimal length below which segments are stiff enough to remain undeformed.

We would like to emphasize that the construction of the floppy modes only relies on the presence of the mesoscopic length $l_{f}$, which is applicable to a broad class of networks. In the particular case of a random rod network we have found that the anomalous scaling properties of the shear modulus, previously found in the simulations, crucially depend on the presence of a second length scale $l_{\text {min }}$, which is a special property of this random architecture. The exponents found for random rod networks are therefore not immediately applicable to other systems. Having established the general theoretical framework, it is nevertheless straightforward to calculate the exponents for other types of networks in two and three dimensions. Indeed, we have applied the theory to reconstituted actin networks cross-linked and bundled with fas- cin, and found that the calculated exponents are in good agreement with the experimental results [27].

Finally, we also conducted simulations probing the nonlinear elasticity of the random fibrous network as well as modified the network structure by introducing kinks in the contour of the polymers. The results confirm the governing role of the identified length scales and firmly establish that the nonaffine floppy mode picture captures the essential physics of stiff polymer networks similar in spirit to affine rubber elasticity for flexible polymer gels.

In view of this conceptual analogy, the next step could be to assess the importance of cross-link fluctuations, which have been neglected here (as in classical rubber elasticity). By greatly reducing the number of fluctuating degrees of freedom to one per fiber (namely $\delta z$ ), the theory developed here may very well provide a new starting point for the analysis of the statistical mechanics of stiff polymer networks.

\section{ACKNOWLEDGMENTS}

We gratefully acknowledge fruitful discussions with Mark Bathe and Camilla Mohrdieck. Financial support from the German Science Foundation (SFB 486) and the German Excellence Initiative via the program "Nanosystems Initiative Munich (NIM)" is gratefully acknowledged.

\section{APPENDIX: SOLUTION OF EQ. (11) FOR VARIOUS NETWORK STRUCTURES}

In this Appendix, we provide some technical details on how to solve Eq. (11) for various network architectures in two spatial dimensions. Assuming harmonic energies $W(x)$ $=k x^{2} / 2$ we rewrite Eq. (11) symbolically as

$$
k=\left\langle f\left(k, n_{\mathrm{cl}} ;\left\{z_{i}, \theta_{i}\right\}\right)\right\rangle,
$$

where the function $f$ is defined by

$$
f=\min _{y(z)}\left[\frac{2 W_{b}}{\delta z^{2}}+k \sum_{i=1}^{n_{\mathrm{cl}}} \sin ^{2}\left(\theta_{i}\right)\left(\frac{y\left(z_{i}\right)}{\delta z}+\cot \theta_{i}\right)^{2}\right],
$$

and we used Eq. (2) to substitute $\bar{y}_{i}=-\cot \theta_{i} \delta z$.

The network structure enters Eqs. (A1) and (A2) via the variables $\left\{z_{i}, \theta_{i}\right\}$, which relate to the locations $z_{i}$ of the crosslinks on the backbone of the primary fiber as well as the angles $\theta_{i}$ between primary and secondary fibers. The ensemble average $\langle$.$\rangle can then be defined by the probability$ distributions $P\left(\left\{\theta_{i}\right\}\right)$ and $P\left(\left\{l_{i}\right\}\right)$, where segment lengths are given by $l_{i}=z_{i+1}-z_{i}$.

To illustrate the importance of structural features on the elastic properties of the network we solve Eq. (A2) for two types of distributions, relating to random and regular structures, respectively. The random network is characterized by probability distributions as given in Eqs. (13) and (14). The regular network has only one segment length $l_{0}=l_{f} /\left(n_{\mathrm{cl}}-1\right)$ and an angular distribution similar to Eq. (13) but restricted to the interval $\left[\theta_{\min }, \pi-\theta_{\min }\right]$.

For a given realization of the randomness the function $f$ is calculated by performing the minimization with respect to 
the contour $y(z)$. This is achieved in two steps, where first the bending energy $W_{b}[y]$ is minimized for a given set of values $\left\{y\left(z_{i}\right)\right\}$. As explained in the main text, this is equivalent to a cubic spline interpolation. The second step consists of a minimization with respect to the remaining variables $\left\{y\left(z_{i}\right)\right\}$.

Finally solving Eq. (A1) the fiber stiffness $k^{\star}\left(n_{\mathrm{cl}}\right)$ is determined as a function of the number $n_{\mathrm{cl}}$ of cross-links per fiber. A graphical solution for $n_{\mathrm{cl}}=40$ for various network structures is presented in Fig. 10. The function $\langle f(k)\rangle$ is plotted as a function of $k$. The sought after value $k^{\star}$ is found at the point of intersection with the bisecting curve.

The different curves for a given network structure correspond to ensembles of varying size. They seem to diverge in the limit $k \rightarrow \infty$. In fact, in this limit only the bending energy $W_{b}$ contributes to Eq. (A2) and $y_{i} \approx \bar{y}_{i}$. This may make the averaging procedure ill-defined, for example in the case of Eq. (14) where the segment lengths $l_{s}$ can become arbitrarily small. The resulting segmental bending energy $w_{b} \sim l_{s}^{-3}$ shows a divergence and does not have a well defined average value.

As one can see from Fig. 10 the resulting fiber stiffness $k^{\star}$ very sensitively depends on the randomness in the segment lengths, while cross-link angles only play a minor role. This is made particularly clear by comparing the random-segment and the regular-segment network in terms of the exponent $x$, which is defined by $k^{\star} \sim n_{\mathrm{cl}}^{x}$. As stated in the main text the random network has $x \approx 6$, while for the regular network we find $x \approx 4$. We have shown above that the former result derives from the presence of the length scale $l_{\min }$. In contrast, the latter is simply obtained by calculating the bending energy of $n_{\mathrm{cl}}$ segments each of length $l_{0}, W_{b} \sim n_{\mathrm{cl}} \kappa / l_{0}^{3} \sim n_{\mathrm{cl}}^{4}$.
[1] M. Rubinstein and R. H. Colby, Polymer Physics (Oxford University Press, New York, 2003).

[2] W. Kuhn, Kolloid-Z. 76, 258 (1936); F. T. Wall, J. Chem. Phys. 10, 132 (1941); P. J. Flory and J. Rehner, Jr., J. Chem. Phys. 11, 512 (1943).

[3] A. Bausch and K. Kroy, Nat. Phys. 2, 231 (2006).

[4] B. Wagner, R. Tharmann, I. Haase, M. Fischer, and A. R. Bausch, Proc. Natl. Acad. Sci. U.S.A. 103, 13974 (2006).

[5] R. Tharmann, M. M. A. E. Claessens, and A. R. Bausch, Phys. Rev. Lett. 98, 088103 (2007).

[6] M. L. Gardel, J. H. Shin, F. C. MacKintosh, L. Mahadevan, P. A. Matsudaira, and D. A. Weitz, Science 304, 1301 (2004).

[7] Y. Tseng, B. W. Schafer, S. C. Almo, and D. Wirtz, J. Biol. Chem. 277, 25609 (2002).

[8] K. Kroy and E. Frey, Phys. Rev. Lett. 77, 306 (1996).

[9] F. C. MacKintosh, J. Käs, and P. A. Janmey, Phys. Rev. Lett. 75, 4425 (1995).

[10] C. Heussinger and E. Frey, Phys. Rev. Lett. 96, 017802 (2006).

[11] C. Heussinger and E. Frey, Phys. Rev. E 75, 011917 (2007).

[12] J. H. Shin, M. L. Gardel, L. Mahadevan, P. Matsudaira, and D. A. Weitz, Proc. Natl. Acad. Sci. U.S.A. 101, 9636 (2004).

[13] C. Storm, J. J. Pastore, F. C. MacKintosh, T. C. Lubensky, and P. A. Janmey, Nature (London) 435, 191 (2005).

[14] L. J. Gibson and M. F. Ashby, Cellular Solids: Structure and Properties (Cambridge University Press, Cambridge, UK, 1999).

[15] E. Frey, K. Kroy, J. Wilhelm, and E. Sackmann, in Dynamical Networks in Physics and Biology, edited by G. Forgacs and D. Beysens (Springer, Berlin, 1998), Chap. 9.

[16] J. Wilhelm and E. Frey, Phys. Rev. Lett. 91, 108103 (2003).

[17] D. A. Head, A. J. Levine, and F. C. MacKintosh, Phys. Rev. Lett. 91, 108102 (2003); Phys. Rev. E 68, 061907 (2003).

[18] C. Heussinger and E. Frey, Phys. Rev. Lett. 97, 105501 (2006).

[19] Rigidity Theory and Applications, edited by M. F. Thorpe and
P. M. Duxbury (Kluwer Academic/Plenum Publishers, Dordrecht, 1999).

[20] J. C. Maxwell, Philos. Mag. 27, 27 (1864).

[21] V. S. Deshpande, M. F. Ashby, and N. A. Fleck, Acta Mater. 49, 1035 (2001).

[22] C. R. Calladine, Int. J. Solids Struct. 14, 161 (1978).

[23] S. Pellegrino and C. R. Calladine, Int. J. Solids Struct. 22, 409 (1986).

[24] S. Pellegrino, Int. J. Solids Struct. 30, 3025 (1993).

[25] S. Alexander, Phys. Rep. 296, 65 (1998).

[26] S. J. Winder and K. R. Ayscough, J. Cell. Sci. 118, 651 (2005).

[27] O. Lieleg et al., Phys. Rev. Lett. 99, 088102 (2007).

[28] R. Götter, K. Kroy, E. Frey, M. Barmann, and E. Sackmann, Macromolecules 29, 30 (1996).

[29] J.-P. Collet, H. Shuman, R. E. Ledger, S. Lee, and J. W. Weisel, Proc. Natl. Acad. Sci. U.S.A. 102, 9133 (2005).

[30] J. Stoer and R. Bulirsch, Introduction to Numerical Analysis (Springer, Berlin, 2002).

[31] J. A. Åström, J. P. Mäkinen, M. J. Alava, and J. Timonen, Phys. Rev. E 61, 5550 (2000).

[32] J. A. Åström, S. Saarinen, K. Niskanen, and J. Kurkijärvi, J. Appl. Phys. 75, 2383 (1994).

[33] M. Latva-Kokko and J. Timonen, Phys. Rev. E 64, 066117 (2001).

[34] O. Kallmes and H. Corte, Tappi J. 43, 737 (1960).

[35] M. Bathe, C. Heussinger, M. Claessens, A. Bausch, and E. Frey, e-print arXiv:q-bio.BM/0607040.

[36] C. Heussinger, M. Bathe, and E. Frey, Phys. Rev. Lett. 99, 048101 (2007)

[37] M. M. A. E. Claessens, M. Bathe, E. Frey, and A. R. Bausch, Nat. Mater. 5, 748 (2006).

[38] P. R. Onck, T. Koeman, T. van Dillen, and E. van der Giessen, Phys. Rev. Lett. 95, 178102 (2005).

[39] J. F. Marko and E. D. Siggia, Macromolecules 28, 8759 (1995).

[40] P. A. Janmey, M. E. McCormick, S. Rammensee, J. L. Leight, 
P. C. Georges, and F. C. MacKintosh, Nat. Mater. 6, 48 (2007). [41] A. M. Kraynik and W. E. Warren, in Low Density Cellular Plastics, edited by H. Hilyard and C. Cunningham (Kluwer Academic Publisher, Amsterdam, 1994), Chap. 7.

[42] In three spatial dimensions, additional modes are found that relate to the deflection $\mathbf{y}^{\perp}$ of single cross-links.

[43] The probability of intersection of two fibers with relative angle $\theta$ is proportional to the excluded volume $A=\sin \theta l_{f}^{2} / 2$.

[44] By minimizing the energy, instead of calculating the partition function associated with the variables $\delta z_{\alpha}$, we neglect fluctuations of the cross-link coordinates.

[45] The density $\delta \rho$ is measured relative to the rigidity percolation transition occurring at $\rho_{c} l_{f}=6.7$. 